\title{
Physical training is associated with changes in Nuclear magnetic resonance and morphometrical parameters of the skeletal muscle in senescent mice
}

\author{
C. Zancanaro, ${ }^{1}$ R. Mariotti, ${ }^{1}$ F. Perdoni, ${ }^{2}$ E. Nicolato, ${ }^{1}$ M. Malatesta ${ }^{1}$ \\ ${ }^{1}$ Dipartimento di Scienze Morfologico-Biomediche, Sezione di Anatomia e Istologia, University of Verona; \\ ${ }^{2}$ Laboratorio di Biologia Cellulare, Dipartimento di Biologia Animale, University of Pavia, and Istituto di \\ Genetica Molecolare del CNR, Pavia, Italy
}

(C)2007 European Journal of Histochemistry

The effect of a three-month training period on $\mathrm{T} 2$ relaxation time as well as on myofibre size and type was investigated in the lower limbs of senescent mice. After training, $\mathrm{T} 2$ (which is a magnetic resonance imaging parameter known to increase during acute exercise) was significantly higher in trained mice (36.37 \pm 1.27 vs $37.76 \pm 2.06 \mathrm{~ms}, p=0.003, \mathrm{n}=8$ ), whereas no change was found in non-trained animals $(36.35 \pm 1.02$ vs $36.24 \pm 1.15 \mathrm{~ms}, p=0.278, n=8)$. The percentage of muscle limb area evaluated in vivo on magnetic resonance images before and after the experimental period was unchanged in trained mice $(69.84 \pm 2.50$ vs $70.29 \pm 2.29, p=0.896, \mathrm{n}=3$ ) and decreased in non-trained animals $(72.98 \pm 1.68$ vs $64.62 \pm 2.34, p=0.006, n=3)$. Cross-sectional area of fast and slow myofibres, evaluated on paraffin-embedded samples after immunolabelling for skeletal fast fibre myosin, was lower in non-trained than in trained mice in both gastrocnemius and quadriceps muscle, but no change in slow/fast fibre ratio nor in apoptotic rate was found. These data show that training can prevent sarcopenia in senescent mice by affecting muscle status and inducing myofibre hypertrophy in the absence of significant muscle damage.

Key words: training, ageing, skeletal muscle, magnetic resonance imaging, fibre type, sarcopenia.

Correspondence: Carlo Zanacanaro

Dipartimento di Scienze Morfologico-Biomediche, Sezione di Anatomia e Istologia, Università degli Studi di Verona, Strada Le Grazie, 8

37134 Verona, Italy

Tel: +390458027155.

Fax: +390458027163

E-mail: carlo.zancanaro@univr.it

Paper accepted on November 19, 2007

European Journal of Histochemistry

2007; vol. 51 issue 4 (October-December):305-310
A geing is associated with a progressive decline of muscle mass, strength and quality, a condition overall known as sarcopenia. These age-related changes have been reported even among healthy, physically active subjects and the rate of muscle loss in humans has been estimated to range between 1 and $2 \%$ per year, past the age of 50 (Hughes et al., 2002). Therefore, sarcopenia represents a powerful risk factor for frailty, loss of independence and physical disability in elderly (Roubenoff et al., 2000). In addition to the impairment of motor function, sarcopenia can involve a number of metabolic and physiological consequences which have been only partially investigated: changes in muscle mass can be associated with osteoporosis (Szulc et al., 2005), altered thermoregulation (e.g., decreased thermogenic capacity of muscle due to reduced mass) (Wilson and Morley, 2003) as well as with a decrease in resting metabolic rate secondary to decreased fat-free mass and decreased physical activity, resulting in a higher prevalence of insulin resistance, type 2 diabetes mellitus, dyslipidemia, and hypertension (Karakelides and Sreekumaran Nair, 2005).

The contributing mechanism(s) leading to sarcopenia remain to be fully elucidated; they are probably multifactorial, including among others: denervation and reinnervation of motor units (Ansved and Larsson, 1989), alteration in the hormonal milieu in which anabolic hormone concentrations progressively decline (Tenover, 1997), elevated concentrations of inflammatory mediators (Bruunsgaard et al., 2001), myonuclear loss through apoptotic mechanisms (Dirks and Leeuwenburgh, 2005), loss of satellite cells (Verdijk et al., 2007), decrease in microvascular function and exercise tolerance (Payne and Bearden, 2006).

Understanding the mechanisms which induce to sarcopenia therefore represents an essential step towards the development of effective interventions to contrast muscle loss and physical disability at 
advanced age. At present, physical exercise has been recognized as the most effective activity to prevent or treat age-related muscle wasting (Marcell, 2003).

In this study we investigated the effect of a programmed long-lasting aerobic physical exercise on the skeletal muscle of senescent mice by combining nuclear magnetic resonance (NMR), which provides in vivo information on muscle status, and light microscopy techniques (morphometry and immunohistochemistry) to obtain a quali-quantitative characterization of myofibres.

\section{Materials and Methods}

Sixteen 20 month-old male mice were used in this study: eight mice were trained by treadmill running (45 min at 9.5-14.2 $\mathrm{m} / \mathrm{min}$ five days a week) for three months, while eight had only spontaneous activity in the cage. All animals had free access to chow and water. The muscles chosen for our study were the quadriceps and the gastrocnemius, mostly containing fast, type II fibres and therefore largely affected by sarcopenia (Larsson et al., 1978; Lexell, 1995).

\section{Nuclear magnetic resonance}

Magnetic resonance imaging was performed before and at the end of the three-month experimental period. Mice ( $n=8$ for each experimental group) were anaesthetized with isofluorane and imaged in a Bruker Biospeck tomography equipped with an 0xford $33 \mathrm{~cm}$ bore magnet operating at 4.7 Tesla. Quantitative T2 maps of the lower limb muscles were taken (spin-echo multiecho sequence, FOV $4 \mathrm{~cm}$, slice thickness $2 \mathrm{~mm}$, interslice distance $4 \mathrm{~mm}, \mathrm{TR} 2000 \mathrm{~ms}$, TE $10 \mathrm{~ms}$, matrix size $128 \times 128$, in-plane resolution $0.312 \mathrm{~mm}^{2}$ ). In three animals of either experimental group, 3D imaging of the lower limbs was performed for morphometry: a spin-echo T1 weighted sequence was used (FOV 3,5 $\mathrm{cm}$, slice thickness $40 \mathrm{~mm}$, TR $200 \mathrm{~ms}$ TE $6 \mathrm{~ms}$, matrix size $128 \times 128 \times 64$, in-plane resolution 0,237 $\mathrm{mm} \times 0,237 \mathrm{~mm} \times 0,547 \mathrm{~mm}$ ); eight coronal sections (Figure 1) per animal, taken at given distances from the coxo-femoral joint, (four acquired before and four at the end of the experimental period) were submitted to morphometrical analysis by using the software Image $\mathrm{J}(\mathrm{NIH})$ : the total area of each leg section and the area occupied by the muscle tissue were measured, then the percentage of leg
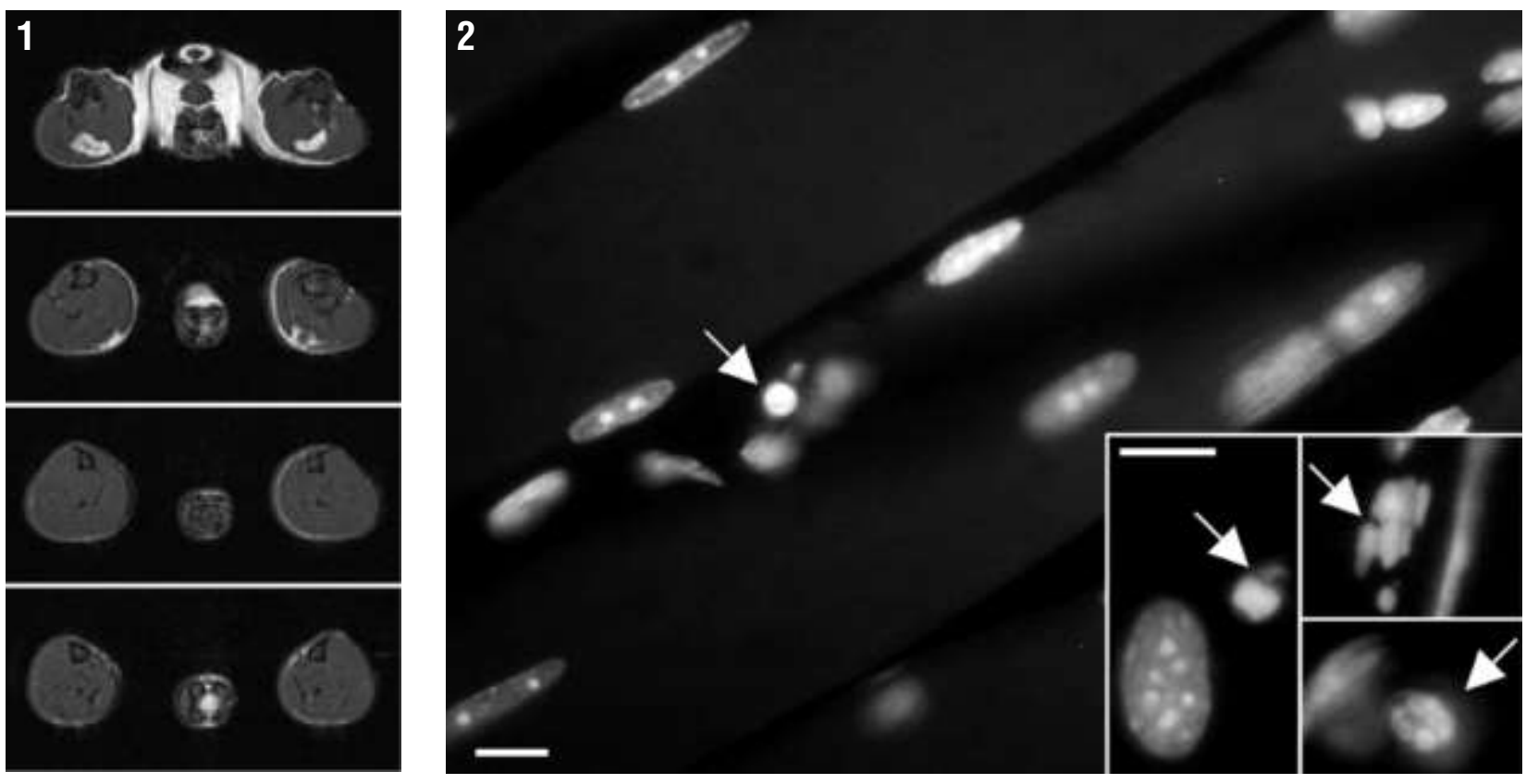

Figure 1. Magnetic resonance imaging of the coronal sections of mouse lower limbs; for quantitative analysis, the percentage of total limb area occupied by the muscle in the four sections was calculated and compared before and after the three-month experimental period.

Figure 2. Quadriceps from a trained mouse, DNA staining with Hoechst 33258: apoptotic nuclei (arrow) are quite scarce. B-D: Apoptotic nuclei (arrows) showing typical chromatin condensation and fragmentation. Bars: $20 \mu \mathrm{m}$. 
sectional surface occupied by muscle was calculated in order to estimate the muscle area independently from the leg dimension.

\section{Light microscopy}

At the end of the experimental period three trained and three non-trained mice were anaesthetized and perfused with $4 \%$ paraformaldehyde in $0.1 \mathrm{M}$ phosphate buffer, $\mathrm{pH}$ 7.4. The gastrocnemius and quadriceps muscles were further fixed by immersion for several hours at $4^{\circ} \mathrm{C}$ in the same fixative solution, then dehydrated with ethanol and embedded in paraffin wax. Five- $\mu \mathrm{m}$-thick transversally sectioned muscle samples were either stained with hematoxylin-eosin or immunolabelled for fibre typing: sections were incubated overnight at $4^{\circ} \mathrm{C}$ with a mouse monoclonal antibody recognizing the heavy chain of the skeletal fast fibre myosin (clone MY-32, Sigma-Aldrich, Buchs, Switzerland), then revealed with an Alexa 488 conjugated antibody against mouse IgG (Molecular Probes, Invitrogen, Milan). The sections were finally counterstained for DNA with $0.1 \mathrm{mg} / \mathrm{mL}$ Hoechst 33258, to detect apoptotic nuclei based on chromatin morphology. Micrographs were taken with an Olympus BX51 microscope equipped with a $100 \mathrm{~W}$ mercury lamp under the following conditions: $330-$ to $385-\mathrm{nm}$ excitation filter (excf), 400-nm dichroic mirror (dm), and 420-nm barrier filter (bf), for Hoechst 33258; 450- to 480-nm excf, 500-nm dm, and 515 $\mathrm{nm}$ bf, for Alexa 488. Images were taken with an Olympus Camedia C-5050 digital camera and stored on a PC by the Olympus software, for processing and printing. Morphometrical evaluation of fibre size was performed on samples immunolabelled for fast myosin (to assess fast and slow fibre size separately): the cross-sectional area of 100 muscle fibres per sample was measured at 40X magnification by using the software Image $J$ (NIH). Moreover, the percentage of fast and slow muscle fibres was calculated on a total of 400 fibres per sample. Finally, the percentage of apoptotic nuclei was evaluated on a total of 1000 cell nuclei per muscle sample.

\section{Statistics}

Results for each variable were pooled according to the experimental groups and the meantstandard error of the mean (SE) values calculated. Statistical comparisons were performed by one-way ANOVA test (the statistical significance was set at $p^{\prime \prime} 0.05$ ).

\section{Results}

\section{Nuclear magnetic resonance}

In lower limb skeletal muscles from trained mice, T2 relaxation time increased significantly ( $36.37 \pm 1.27$ vs $37.76 \pm 2.06 \mathrm{~ms}, p=0.003)$ at the end of the experimental period, whereas in nontrained animals no change was found $(36.35 \pm 1.02$ vs $36.24 \pm 1.15 \mathrm{~ms}, p=0.278$ ).

Morphometrical evaluation of coronal sections of lower limbs revealed similar values for the percentage of muscle area in trained animals before and after the experimental period $(69.84 \pm 2.50$ vs $70.29 \pm 2.29$, respectively, $p=0.896$ ), whereas muscle area significantly decreased in non-trained animals $(72.98 \pm 1.68$ vs $64.62 \pm 2.34, p=0.006)$. The percentage of the muscle area was similar in the two groups before the experimental period $(p=0.303)$.

\section{Light microscopy}

Morphological observation of muscle samples did not reveal necrotic features in trained nor in nontrained mice. Apoptotic nuclei (Figure 2) were quite scarce and their percentage did not differ in the two groups: $0.20 \pm 0.05 \%$ vs $0.25 \pm 0.04 \%$ $(p=0.487)$ and $0.25 \pm 0.06 \%$ vs $0.21 \pm 0.05 \%$ ( $p=0.783$ ) in the quadriceps and in the gastrocnemius muscle, respectively.

Morphometrical analyses demonstrated a significantly smaller myofibre cross-sectional area in nontrained than in trained mice in both gastrocnemius and quadriceps muscle; such a difference was associated with smaller fast and slow fibres, although the size difference was much more evident in the fast fibres (Figure 3A, B). No change in the percentage of slow and fast myofibres was found in either muscle of the two groups (Figure 3C).

\section{Discussion}

Sarcopenia consists in the reduction of skeletal muscle mass, strength and resistance, with consequent impairment of performance in older individuals. It is known that sarcopenia shows a loss of muscle fibres as well as a decrease in total crosssectional area of the remaining myofibres (Lexell, 1995; Brooks and Faulkner, 1994), especially in muscle which mostly contain type II fibres (Larsson et al., 1978; Lexell, 1995). Accordingly, we observed a significant reduction in both muscle mass and myofibre size in non-trained senescent 
mice; in particular, although both fast type II and slow type I myofibres were significantly smaller than those of trained mice, fast fibres showed a higher sensitivity $(30-50 \%$ of size reduction in comparison to $16-17 \%$ in slow fibres) to the sarcopenic process, consistent with previous findings (Vandervoort, 2002; Alway et al., 2003). In the muscles analysed in this study, there was no evidence for the conversion from fast type II fibres to slow type I fibres nor for a clustering of slow fibres; however, we cannot exclude that this phenomenon may occur after longer ageing periods.

Senescent mice submitted to a 3-month training programme did not show any alteration in muscle mass and myofibre size, indicating that a programmed long-lasting aerobic physical exercise can counteract the sarcopenic process. In addition, our data suggest that physical exercise would maintain the senescent muscle in a long-lasting activated condition: in fact, we found a slight but significant increase of $\mathrm{T} 2$ values in trained mice after an overnight rest; previously, increased T2 values in skeletal muscle were reported during acute exercise, representing an index of strong muscle recruitment, intense contraction or high metabolic state (Patten et al., 2003). Although physical exercise increases oxidative stress on skeletal muscle, it appears to have a positive effect on mitochondrial density and functions (Coggan et al., 1992). It is possible that a moderate, daily physical exercise can improve mitochondrial activity without severely increasing free radical generation, thus having a positive effect on the whole muscle metabolism. Moreover, it has been recently reported (Melov et al., 2007) that physical training induces in elderly skeletal muscles a gene transcription pattern quite similar to young individuals, thus demonstrating that physical activity not only affects the whole functional status of the muscles, but also reverts already established age-related changes.

Interestingly, neither necrotic features nor prominent apoptotic rate were observed in both trained and non-trained mice. Apoptotic rate in skeletal muscle has been shown to increase with ageing and disuse as well as with acute exercise, whereas it decreases with physical training; however, the role of apoptosis in sarcopenic muscle fibre loss is still debated (reviews in Dirks, 2005; DupontVersteegden, 2005). In this work the training protocol used did not induce cell damage or death in the presence of maintained muscle mass, thereby suggesting that moderate, constant physical activity is beneficial to sarcopenia; on the other hand, spontaneous motor activity could not prevent sarcopenia, albeit increase of necrotic or apoptotic processes in myofibres was not found.

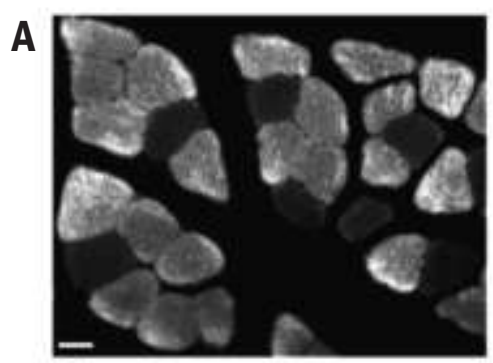

C

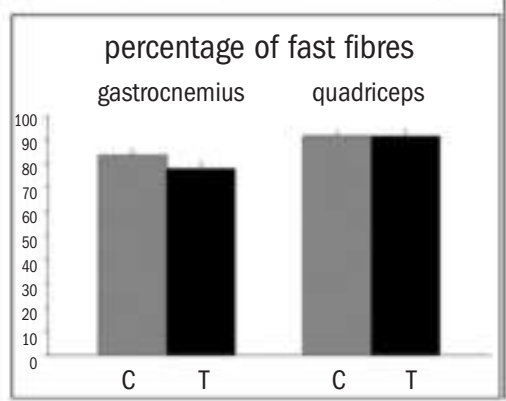

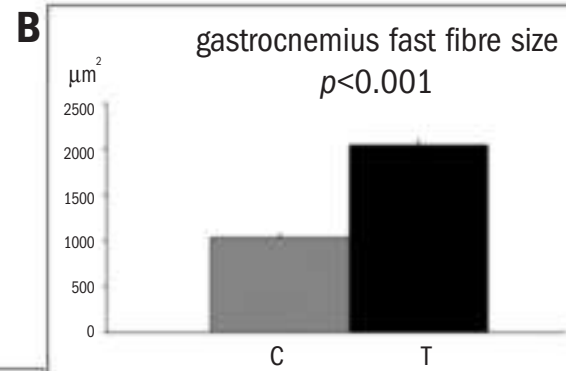

gastrocnemius slow fibre size $p=0.021$

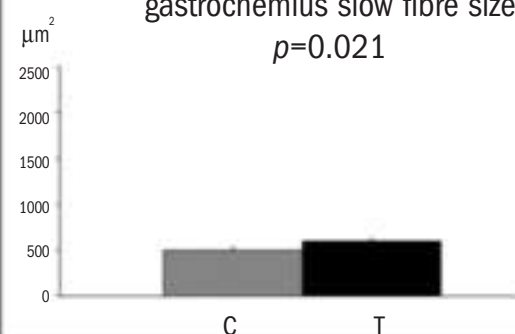

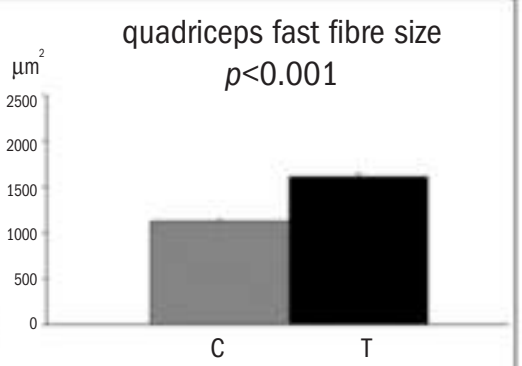

quadriceps slow fibre size $p=0.021$

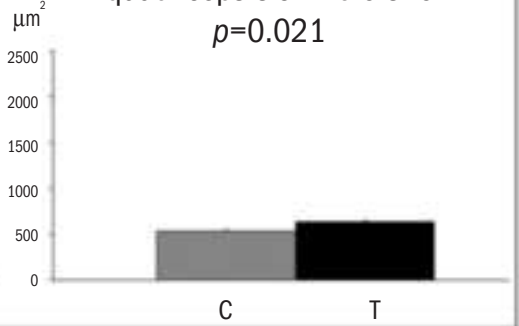

Figure 3. Immunolabelled fast type II fibres in the gastrocnemius of a trained mouse. Bar: 20 um. B. Both fast and slow fibres of the gastrocnemius and the quadriceps show significantly smaller size in non-trained (control, $C$ ) than in trained ( $T$ ) mice. $C$. The percentage of fast fibres does not change between non-trained (control, $C$ ) and trained $(T)$ mice in both the gastrocnemius $(p=0.233)$ and the quadriceps $(p=0.949)$. 


\section{References}

Alway SE, Degens H, Krishnamurthy G, Chaudhrai A. Denervation stimulates apoptosis but not Id2 expression in hindlimb muscles of aged rats. J Gerontol A Biol Sci Med Sci 2003;58:687-97.

Ansved T, Larsson L. Effects of ageing on enzyme-histochemical, morphometrical and contractile properties of the soleus muscle in the rat. J Neurol Sci 1989;93:105-24.

Brooks SV, Faulkner JA. Skeletal muscle weakness in old age: underlying mechanisms. Med Sci Sports Exerc 1994; 26:432-9.

Bruunsgaard H, Pedersen M, Pedersen BK. Aging and proinflammatory cytokines. Curr Opin Hematol 2001;8:131-6.

Coggan AR, Spina RJ, King DS, Rogers MA, Brown M, Nemeth PM, Holloszy J0. Skeletal muscle adaptations to endurance training in 60- to 70-yr-old men and women. J Appl Physiol 1992;72:1780-6.

Dirks A, Leeuwenburgh C. The role of apoptosis in age-related skeletal muscle atrophy. Sports Med 2005; 35:473-83.

Dupont-Versteegden EE. Apoptosis in muscle atrophy: relevance to sarcopenia. Exp Gerontol 2005; 40:473-81.

Hughes VA, Frontera WR, Roubenoff $R$, Evans WJ, Singh MA. Longitudinal changes in body composition in older men and women: role of body weight change and physical activity. Am J Clin Nutr 2002;76:473-81.

Karakelides H, Sreekumaran Nair K. Sarcopenia of aging and its metabolic impact. Curr Top Dev Biol 2005; 68:123-48.

Larsson L, Sjodin B, Karlsson J. Histochemical and biochemical changes in human skeletal muscle with age in sedentary males, ages
22-65 years. Acta Physiol Scand 1978; 103:31-9.

Lexell J. Human aging, muscle mass, and fibre type composition. J Gerontol A Biol Sci Med Sci 1995; 50:11-6.

Marcell TJ. Sarcopenia: causes, consequences, and preventions. J Gerontol A Biol Sci Med Sci 2003; 58:M911-6.

Melov S, Tarnopolsky MA, Beckman K, Felkey K, Hubbard A. Resistance exercise reverses aging in human skeletal muscle. PLoS ONE 2007; 2:e465.

Patten C, Meyer RA, Fleckenstein JL. T2 mapping of muscle. Semin Musculoskelet Radiol 2003; 7:297-305.

Payne GW, Bearden SE. The microcirculation of skeletal muscle in aging. Microcirculation 2006; 13:275-7.

Roubenoff R. Sarcopenia and its implications for the elderly. Eur J Clin Nutr 2000; 54 Suppl 3:S40-7.

Szulc P, Beck TJ, Marchand F, Delmas PD. Low skeletal muscle mass is associated with poor structural parameters of bone and impaired balance in elderly men-the MINOS study. J Bone Miner Res 2005; 20:721-9.

Tenover JL. Testosterone and the aging male. J Androl 1997; 18:103-6.

Vandervoort AA. Aging of the human neuromuscular system. Muscle Nerve 2002; 25:17-25.

Verdijk LB, Koopman R, Schaart G, Meijer K, Savelberg HH, van Loon LJ. Satellite cell content is specifically reduced in type II skeletal muscle fibers in the elderly. Am J Physiol Endocrinol Metab 2007; 292:E151-7.

Wilson MM, Morley JE. Invited review: Aging and energy balance. J Appl Physiol 2003; 95:1728-36. 
C. Zancanaro et al. 